TRANSACTIONS OF THE

AMERICAN MATHEMATICAL SOCIETY

Volume 360, Number 5, May 2008, Pages 2423-2442

S 0002-9947(07)04389-9

Article electronically published on December 11, 2007

\title{
FRACTAL HAMILTON-JACOBI-KPZ EQUATIONS
}

\author{
GRZEGORZ KARCH AND WOJBOR A. WOYCZYŃSKI \\ Dedicated to our friend and collaborator, Piotr Biler
}

\begin{abstract}
Nonlinear and nonlocal evolution equations of the form $u_{t}=\mathcal{L} u \pm$ $|\nabla u|^{q}$, where $\mathcal{L}$ is a pseudodifferential operator representing the infinitesimal generator of a Lévy stochastic process, have been derived as models for growing interfaces in the case when the continuous Brownian diffusion surface transport is augmented by a random hopping mechanism. The goal of this paper is to study properties of solutions to this equation resulting from the interplay between the strengths of the "diffusive" linear and "hyperbolic" nonlinear terms, posed in the whole space $\mathbb{R}^{N}$, and supplemented with nonnegative, bounded, and sufficiently regular initial conditions.
\end{abstract}

\section{INTRODUCTION}

The well-known Kardar-Parisi-Zhang (KPZ) equation $h_{t}=\nu \Delta h+\frac{\lambda}{2}|\nabla h|^{2}$ was derived in [18] as a model for growing random interfaces. Recall that the interface is parameterized here by the transformation $\Sigma(t)=(x, y, z=h(x, y, t))$, so that $h=h(x, y, t)$ is the surface elevation function, $\nu>0$ is identified in [18] as a "surface tension" or "high diffusion coefficient", $\Delta$ and $\nabla$ stand, respectively, for the usual Laplacian and gradient differential operators in spatial variables, and $\lambda \in \mathbb{R}$ scales the intensity of the ballistic rain of particles onto the surface.

An alternative, first-principles derivation of the KPZ equation (cf. [20], for more detailed information and additional references) makes three points:

(a) The Laplacian term can be interpreted as a result of the surface transport of adsorbed particles caused by the standard Brownian diffusion.

Received by the editors January 27, 2006.

2000 Mathematics Subject Classification. Primary 35K55, 35B40, 60H30.

Key words and phrases. Nonlinear evolution equation, Lévy anomalous diffusion, self-similar asymptotics, surface transport.

The authors appreciate valuable comments of the referee which helped them to improve the original version of this paper. Also, they would like to thank J. Droniou for making his unpublished manuscript available to them. This paper was partially written while the first-named author enjoyed the hospitality and support of the Center for Stochastic and Chaotic Processes in Science and Technology at Case Western Reserve University, Cleveland, Ohio, sponsored by the U.S. National Science Foundation Grant INT-0310055, and of the Helsinki University of Technology, and the University of Helsinki, Finland, within the Finnish Mathematical Society Visitor Program in Mathematics 2005-2006, Function Spaces and Differential Equations. The preparation of this paper was also partially supported by the KBN grant 2/P03A/002/24, and by the European Commission Marie Curie Host Fellowship for the Transfer of Knowledge "Harmonic Analysis, Nonlinear Analysis and Probability" MTKD-CT-2004-013389.

(C) 2007 American Mathematical Society Reverts to public domain 28 years from publication 
(b) In several experimental situations a hopping mechanism of surface transport is present which necessitates augmentation of the Laplacian by a nonlocal term modeled by a Lévy stochastic process.

(c) The quadratic nonlinearity is a result of truncation of a series expansion of a more general, physically justified, nonlinear even function.

These observations lead us to consider in this paper a nonlinear nonlocal equation of the form

$$
u_{t}=-\mathcal{L} u+\lambda|\nabla u|^{q},
$$

where the Lévy diffusion operator $\mathcal{L}$ is defined as

$\mathcal{L} v(x)=-\sum_{j, k=1}^{N} Q_{j, k} \frac{\partial^{2} v(x)}{\partial x_{j} \partial x_{k}}+\int_{\mathbb{R}^{N}}\left(v(x)-v(x+y)+y \cdot \nabla v(x) \mathbb{I}_{\{|y|<1\}}(y)\right) \Pi(d y)$.

The matrix $\left\{Q_{j, k}\right\}_{j, k=1}^{N}$ in (1.2) is assumed to be nonnegative-definite; if it is not degenerate, a linear change of the variables transforms the first term in (1.2) into the usual Laplacian $-\Delta$ on $\mathbb{R}^{N}$ which corresponds to the Brownian part of the diffusion modeled by $\mathcal{L}$. The second term on the right-hand side of (1.2) models the hopping phenomena and is determined by the Borel measure $\Pi$, usually called the Lévy measure of the stochastic process, such that $\Pi(\{0\})=0$, and $\int_{\mathbb{R}^{N}} \min \left(1,|y|^{2}\right) \Pi(d y)<\infty$. One could also include on the right-hand side a drift term $b \cdot \nabla v$, where $b \in \mathbb{R}^{N}$ is a fixed vector but, for the sake of the simplicity of the exposition, we omit it. All necessary assumptions and properties of Lévy diffusion operators, as well as the semigroups of linear operators generated by $-\mathcal{L}$, are gathered at the beginning of the next section.

Relaxing the assumptions that led to the quadratic expression in the classical KPZ equation, the nonlinear term in (1.1) has the form

$$
\lambda|\nabla u|^{q}=\lambda\left(\left|\partial_{x_{1}} u\right|^{2}+\ldots+\left|\partial_{x_{N}} u\right|^{2}\right)^{q / 2},
$$

where $q$ is a constant parameter. To study the interaction of the "strength" of the nonlocal Lévy diffusion parameterized by the Lévy measure $\Pi$, with the "strength" of the nonlinear term, parameterized by $\lambda$ and $q$, we consider in (1.1) the whole range, $1<q<\infty$, of the nonlinearity exponent.

Finally, as far as the intensity parameter $\lambda \in \mathbb{R}$ is concerned, we distinguish two cases:

- The deposition case: Here, $\lambda>0$ characterizes the intensity of the ballistic deposition of particles on the evolving interface.

- The evaporation case: Here, $\lambda<0$, and the model displays a time-decay of the total "mass" $M(t)=\int_{\mathbb{R}^{N}} u(x, t) d x$ of the solution (cf. Proposition 3.5).

Equation (1.1) will be supplemented with the nonnegative initial datum,

$$
u(x, 0)=u_{0}(x),
$$

and our standing assumptions are that $u_{0} \in W^{1, \infty}\left(\mathbb{R}^{N}\right)$, and $u_{0}-K \in L^{1}\left(\mathbb{R}^{N}\right)$, for some constant $K \in \mathbb{R}$; as usual, $W$, with some superscripts, stands for various Sobolev spaces.

The long-time behavior of solutions to the viscous Hamilton-Jacobi equation $u_{t}=\Delta u+\lambda|\nabla u|^{q}$, with $\lambda \in \mathbb{R}$, and $q>0$, has been studied by many authors; see e.g. $[1,2,3,4,11,19]$, and the references therein. The dynamics of solutions to 
this equation is governed by two competing effects, one resulting from the diffusive term $\Delta u$, and the other corresponding to the "hyperbolic" nonlinearity $|\nabla u|^{q}$. The above-cited papers aimed at explaining how the interplay of these two effects influences the large-time behavior of solutions depending on the values of $q$ and the initial data. The present paper follows that strategy as well. Hence, we want to understand the interaction of the diffusive nonlocal Lévy operator (1.2) with the power-type nonlinearity. Our results can be viewed as extensions of some of the above-quoted work. However, their physical context is quite different and, to prove them, new mathematical tools have to be developed.

For the sake of completeness we mention other recent works on nonlinear and nonlocal evolution equations. First, note that equation (1.1) also often appears in the context of optimal control of jump diffusion processes. Here, the theory of the viscosity solutions provides a good framework to study these equations. We refer the reader to the works of Jakobsen and Karlsen [14, 15], and Droniou and Imbert $[12,9]$ for more detailed information and references. Fractional conservation laws, including the fractional Burgers equation, were studied in $[5,16,17,21]$ via probabilistic techniques such as nonlinear McKean processes and interacting diffusing particle systems.

In the next section, we specify our assumptions on the Lévy diffusion operator and state the main results concerning the nonlinear problem (1.1)-(1.3). Section 3 contains proofs of those results which are independent of the sign of the intensity parameter $\lambda$ : the existence of solutions, the maximum principle, and the decay of $\|\nabla u(t)\|_{p}$ for certain $p>1$. Further properties of solutions to (1.1)-(1.3) in the deposition case $\lambda>0$ are studied in Section 4. Properties specific for the evaporation case $\lambda<0$ appear in Section 5. Finally, the self-similar asymptotics of the solutions is derived in Section 6 .

Standard notation is used throughout the paper. For $1 \leq p \leq \infty$, the $L^{p}$-norm of a Lebesgue measurable, real-valued function $v$ defined on $\mathbb{R}^{N}$ is denoted by $\|v\|_{p}$. The set $C_{b}(\Omega)$ consists of continuous and bounded functions on $\Omega$, and $C_{b}^{k}(\Omega)$ contains functions with $k$ bounded derivatives. The space of rapidly decaying, real-valued functions is denoted by $\mathcal{S}\left(\mathbb{R}^{N}\right)$. The Fourier transform of $v$ is $\widehat{v}(\xi) \equiv$ $(2 \pi)^{-N / 2} \int_{\mathbb{R}^{N}} e^{-i x \xi} v(x) d x$. The constants independent of solutions and of $t$ (but, perhaps, dependent on the initial values) will be denoted by the same letter $C$, even if they may vary from line to line. Occasionally, we write, e.g., $C=C(\alpha, \ell)$ when we want to emphasize the dependence of $C$ on parameters $\alpha$, and $\ell$.

\section{MAin RESUlts AND COMmEnts}

We begin by gathering basic properties of solutions of the linear Cauchy problem

$$
u_{t}=-\mathcal{L} u, \quad u(x, 0)=u_{0}(x),
$$

where the symbol $a=a(\xi)$ of the pseudodifferential operator $\mathcal{L}$ has the LévyKhintchine representation (cf. [13, Chapter 3])

$$
a(\xi)=Q \xi \cdot \xi+\int_{\mathbb{R}^{n}}\left(1-e^{-i \eta \xi}-i \eta \cdot \xi \mathbb{I}_{\{|\eta|<1\}}(\eta)\right) \Pi(d \eta) .
$$

For every $v \in \mathcal{S}\left(\mathbb{R}^{N}\right)$, one can use formula (2.2) to invert the Fourier transform $\widehat{\mathcal{L} v}(\xi)=a(\xi) \widehat{v}(\xi)$ and to get representation (1.2). In view of (1.2), one can show (cf. [13, Thm. 4.5.13]) that $\mathcal{L} v$ is well defined for every $v \in C_{b}^{2}\left(\mathbb{R}^{N}\right)$. 
It is well-known that the operator $-\mathcal{L}$ generates a positivity-preserving, symmetric Lévy semigroup $e^{-t \mathcal{L}}$ of linear operators on $L^{1}\left(\mathbb{R}^{n}\right)$ of the form

$$
\left(e^{-t \mathcal{L}} v\right)(x)=\int_{\mathbb{R}^{N}} v(x-y) \mu^{t}(d y),
$$

where the family $\left\{\mu^{t}\right\}_{t \geq 0}$ of probability Borel measures on $\mathbb{R}^{N}$ (called the convolution semigroup in [13]) satisfies $\widehat{\mu^{t}}(\xi)=(2 \pi)^{-N / 2} e^{-t a(\xi)}$. For every $1<p<\infty$, the semigroup $e^{-t \mathcal{L}}$ is analytic on $L^{p}\left(\mathbb{R}^{n}\right)$; cf. [13, Thm. 4.2.12]. Moreover, the representation (2.3), and the properties of the measures $\mu^{t}$, imply that if $0 \leq v \leq 1$, almost everywhere, then $0 \leq e^{-t \mathcal{L}} v \leq 1$, almost everywhere (i.e., $e^{-t \mathcal{L}}$ is a subMarkovian semigroup on $\left.L^{p}\left(\mathbb{R}^{N}\right)\right)$.

The basic assumption throughout the paper is that the Lévy operator $\mathcal{L}$ is a "perturbation" of the fractional Laplacian $(-\Delta)^{\alpha / 2}$, or, more precisely, that it satisfies the following condition:

- The symbol $a$ of the operator $\mathcal{L}$ can be written in the form

$$
a(\xi)=\ell|\xi|^{\alpha}+k(\xi),
$$

where $\ell>0, \alpha \in(0,2]$, and the pseudodifferential operator $\mathcal{K}$, corresponding to the symbol $k$, generates a strongly continuous semigroup of operators on $L^{p}\left(\mathbb{R}^{N}\right), 1 \leq p \leq \infty$, with norms uniformly bounded in $t$.

Observe that, without loss of generality (rescaling the spatial variable $x$ ), we can assume that the scaling constant $\ell$ in (2.4) is equal to 1 . Also, note that the above assumptions on the operator $\mathcal{K}$ are satisfied if the Fourier transform of the function $e^{-t k(\xi)}$ is in $L^{1}\left(\mathbb{R}^{N}\right)$, for every $t>0$, and its $L^{1}$-norm is uniformly bounded in $t$.

The study of the large time behavior of solutions to the nonlinear problem (1.1)(1.3) will necessitate the following supplementary asymptotic condition on $\mathcal{L}$ :

- The symbol $k=k(\xi)$ appearing in (2.4) satisfies the condition

$$
\lim _{\xi \rightarrow 0} \frac{k(\xi)}{|\xi|^{\alpha}}=0 .
$$

The assumptions (2.4) and (2.5) are fulfilled, e.g., by multifractional diffusion operators

$$
\mathcal{L}=-a_{0} \Delta+\sum_{j=1}^{k} a_{j}(-\Delta)^{\alpha_{j} / 2},
$$

with $a_{0} \geq 0, a_{j}>0,1<\alpha_{j}<2$, and $\alpha=\min _{1 \leq j \leq k} \alpha_{j}$, but, more generally, one can consider here

$$
\mathcal{L}=(-\Delta)^{\alpha / 2}+\mathcal{K}
$$

where $\mathcal{K}$ is a generator of another Lévy semigroup. Nonlinear conservation laws with such nonlocal operators were studied in $[6,7,8]$.

In view of the assumption (2.4) imposed on its symbol $a(\xi)$, the semigroup $e^{-t \mathcal{L}}$ satisfies the following decay estimates (cf. [8, Sec. 2], for details):

$$
\begin{aligned}
\left\|e^{-t \mathcal{L}} v\right\|_{p} & \leq C t^{-N(1-1 / p) / \alpha}\|v\|_{1}, \\
\left\|\nabla e^{-t \mathcal{L}} v\right\|_{p} & \leq C t^{-N(1-1 / p) / \alpha-1 / \alpha}\|v\|_{1},
\end{aligned}
$$

for each $p \in[1, \infty]$, all $t>0$, and a constant $C$ depending only on $p$ and $N$. The sub-Markovian property of $e^{-t \mathcal{L}}$ implies that, for every $p \in[1, \infty]$,

$$
\left\|e^{-t \mathcal{L}} v\right\|_{p} \leq\|v\|_{p}
$$


Moreover, for each $p \in[1, \infty]$, we have

$$
\left\|\nabla e^{-t \mathcal{L}} v\right\|_{p} \leq C t^{-1 / \alpha}\|v\|_{p} .
$$

Let us also note that under the assumption (2.5), the large time behavior of $e^{-t \mathcal{L}}$ is described by the fundamental solution of the linear equation $u_{t}+(-\Delta)^{\alpha / 2} u=0$. This result is recalled below in Lemma 6.1.

We are now in a position to present our results concerning the nonlinear problem (1.1)-(1.3) starting with the fundamental problems of the existence, the uniqueness, and the regularity of solutions. Note that at this stage no restrictions are imposed on the sign of the parameter $\lambda$ and the initial datum $u_{0}$. Consequently, all results of Theorem 2.1 are valid for both the deposition and the evaporation cases.

Theorem 2.1. Assume that the symbol $a=a(\xi)$ of the Lévy operator $\mathcal{L}$ satisfies condition (2.4) with an $\alpha \in(1,2]$. Then, for every $u_{0} \in W^{1, \infty}\left(\mathbb{R}^{N}\right), \lambda \in \mathbb{R}$, and $T>0$, problem (1.1)-(1.3) has the unique solution $u$ in the space $\mathcal{X}=$ $C\left([0, T), W^{1, \infty}\left(\mathbb{R}^{N}\right)\right)$.

If, additionally, there exists a constant $K \in \mathbb{R}$ such that $u_{0}-K \in L^{1}\left(\mathbb{R}^{N}\right)$, then

$$
u-K \in C\left([0, T], L^{1}\left(\mathbb{R}^{N}\right)\right) \quad \text { and } \sup _{0<t \leq T} t^{1 / \alpha}\|\nabla u(t)\|_{1}<\infty .
$$

Moreover, for all $t \geq 0$,

$$
\|u(t)\|_{\infty} \leq\left\|u_{0}\right\|_{\infty} \quad \text { and } \quad\|\nabla u(t)\|_{\infty} \leq\left\|\nabla u_{0}\right\|_{\infty},
$$

and the following comparison principle is valid: for any two initial data satisfying the condition that for all $x \in \mathbb{R}^{N}, u_{0}(x) \leq \tilde{u}_{0}(x)$, the corresponding solutions satisfy the inequality $u(x, t) \leq \tilde{u}(x, t)$, for all $x \in \mathbb{R}^{N}$, and $t \geq 0$.

Remark 2.2. Note that if $u$ is a solution to (1.1), then so is $u-K$, for any constant $K \in \mathbb{R}$. Hence, without loss of generality, in what follows we will assume that $K=0$.

In a recent publication, Droniou and Imbert [9] study a nonlinear-nonlocal viscous Hamilton-Jacobi equation of the form

$$
u_{t}+(-\Delta)^{\alpha / 2} u+F(t, x, u, \nabla u)=0 .
$$

For $\alpha \in(0,2)$, and under very general assumptions on the nonlinearity, they construct a unique, global-in-time viscosity solution for initial data from $W^{1, \infty}\left(\mathbb{R}^{N}\right)$, and emphasize (cf. [9, Remark 5]) that an analogous result can be obtained in the case of more general nonlocal operators, including those given by formula (1.2). That unique solution also satisfies the maximum principle (cf. [9, Thm. 3 and Thm. 5]) which implies inequalities (2.11), and the comparison principle contained in Theorem 2.1. Thus, the $L^{1}$-property of solutions stated in (2.10) (under the additional assumption $u_{0}-K \in L^{1}\left(\mathbb{R}^{N}\right)$ ) is the only truly new result in Theorem 2.1 and, as a result, only this part of Theorem 2.1 will be proved later on (see Section 3 ). Here we only mention that our reasoning involves the integral (mild) equation

$$
u(t)=e^{-t \mathcal{L}} u_{0}+\lambda \int_{0}^{t} e^{-(t-\tau) \mathcal{L}}|\nabla u(\tau)|^{q} d \tau,
$$

estimates (2.11), and the Banach fixed point argument.

Once the global-in-time solution $u$ is constructed, it is natural to ask questions about its behavior as $t \rightarrow \infty$. From now on, equation (1.1) will be supplemented with the nonnegative integrable initial datum (1.3). In view of Theorem 2.1, the 
standing assumption $u_{0} \in W^{1, \infty}\left(\mathbb{R}^{N}\right) \cap L^{1}\left(\mathbb{R}^{N}\right)$ allows as to define the "mass" of the solution to (1.1)-(1.3) by the formula

$$
M(t)=\|u(t)\|_{1}=\int_{\mathbb{R}^{N}} u(x, t) d x, \quad t \geq 0 .
$$

Its large-time behavior is one of the principal objects of study in this paper. It turns out that in the deposition case, i.e., for $\lambda>0$, the function $M(t)$ is nondecreasing in $t$ (cf. Proposition 3.5, below) and, for sufficiently small $q$, escapes to $+\infty$, as $t \rightarrow \infty$. More precisely, we have the following result which is an immediate consequence of the lower bounds for $M(t)$ obtained below in Theorem 4.1.

Theorem 2.3. Let $\lambda>0,1<q \leq \frac{N+\alpha}{N+1}$, and suppose that the symbol a of the Lévy operator $\mathcal{L}$ satisfies conditions (2.4) and (2.5) with $\alpha \in(1,2]$. If $u=u(x, t)$ is a solution to (1.1) with an initial datum satisfying conditions $0 \leq u_{0} \in L^{1}\left(\mathbb{R}^{N}\right) \cap$ $W^{1, \infty}\left(\mathbb{R}^{N}\right)$, and $u_{0} \neq 0$, then $\lim _{t \rightarrow \infty} M(t)=+\infty$.

When $q$ is greater than the critical exponent $(N+\alpha) /(N+1)$, we are able to show that, for sufficiently small initial data, the mass $M(t)$ is uniformly bounded in time.

Theorem 2.4. Let $\lambda>0, q>\frac{N+\alpha}{N+1}$, and suppose that the symbol a of the Lévy operator $\mathcal{L}$ satisfies conditions (2.4) and (2.5) with $\alpha \in(1,2]$. If, either $\left\|u_{0}\right\|_{1}$ or $\left\|\nabla u_{0}\right\|_{\infty}$ is sufficiently small, then $\lim _{t \rightarrow \infty} M(t)=M_{\infty}<\infty$.

Remark 2.5. If we limit ourselves to $\mathcal{L}=(-\Delta)^{\alpha / 2}$ in Theorem 2.4, it suffices only to assume that the quantity $\left\|u_{0}\right\|_{1}\left\|\nabla u_{0}\right\|_{\infty}^{(q(N+1)-\alpha-N) /(\alpha-1)}$ is small which, for $\alpha=2$, is in perfect agreement with the assumption imposed in [19]. To see this fact, note that, for every $R>0$, the equation $u_{t}=-(-\Delta)^{\alpha / 2} u+\lambda|\nabla u|^{q}$ is invariant under rescaling $u_{R}(x, t)=R^{b} u\left(R x, R^{\alpha} t\right)$, with $b=(\alpha-q) /(q-1)$. Choosing $R=$ $\left\|\nabla u_{0}\right\|_{\infty}^{-1 /(1+b)}$ we immediately obtain $\left\|\nabla u_{0, R}\right\|_{\infty}=R^{1+b}\left\|\nabla u_{0}\right\|_{\infty}=1$. Hence, the conclusion follows from the smallness assumption imposed on $\left\|u_{0, R}\right\|_{1}$ in Theorem 2.4 and from the identity $\left\|u_{0, R}\right\|_{1}=\left\|u_{0}\right\|_{1} R^{b-n}=\left\|u_{0}\right\|_{1}\left\|\nabla u_{0}\right\|_{\infty}^{(q(N+1)-\alpha-N) /(\alpha-1)}$.

If the Lévy operator $\mathcal{L}$ has a nondegenerate Brownian part, and if $q \geq 2$, we can strengthen the assertion of Theorem 2.4 and show that the mass of every solution (not necessary small) is bounded as $t \rightarrow \infty$.

Theorem 2.6. Let $\lambda>0, q \geq 2$, and suppose that the Lévy diffusion operator $\mathcal{L}$ has a nondegenerate Brownian part. Then, each nonnegative solution to (1.1)-(1.3) with an initial datum $u_{0} \in W^{1, \infty}\left(\mathbb{R}^{N}\right) \cap L^{1}\left(\mathbb{R}^{N}\right)$ has the mass $M(t)=\int_{\mathbb{R}^{N}} u(x, t) d x$ increasing to a finite limit $M_{\infty}$, as $t \rightarrow \infty$.

Remark 2.7. The smallness assumption imposed in Theorem 2.4 seems to be necessary. Indeed, for $\mathcal{L}=-\Delta$, it is known that if $\lambda>0$, and $(N+2) /(N+1)<q<2$, then there exists a solution to (1.1)-(1.3) such that $\lim _{t \rightarrow \infty} M(t)=+\infty$ (cf. [4] and [2, Thm. 2.4]). Moreover, if $\left\|u_{0}\right\|_{1}$ and $\left\|\nabla u_{0}\right\|_{\infty}$ are "large", then the large-time behavior of the corresponding solution is dominated by the nonlinear term ([2]), and one can expect that $M_{\infty}=\infty$. We conjecture that analogous results hold true at least for the $\alpha$-stable operator (fractional Laplacian) $\mathcal{L}=(-\Delta)^{\alpha / 2}$, and for $q$ satisfying the inequality $(N+\alpha) /(N+1)<q<\alpha$. We also conjecture that the critical exponent $q=2$, for $\mathcal{L}=-\Delta$, should be replaced by $q=\alpha$ if $\mathcal{L}$ has a nontrivial $\alpha$-stable part. In this case, for $q \geq \alpha$, we expect that, as $t \rightarrow \infty$, the mass 
of any nonnegative solution converges to a finite limit, just like in Theorem 2.6. Our expectation is that the proof of this conjecture can be based on a reasoning similar to that contained in the proof of Theorem 2.6. However, at this time, we were unable to obtain those estimates in a more general case.

In the evaporation case, $\lambda<0$, the mass $M(t)$ is a nonincreasing function of $t$ (cf. Proposition 3.5, below), and the question, answered in the next two theorems, is when it decays to 0 and when it decays to a positive constant.

Theorem 2.8. Let $\lambda<0,1 \leq q \leq \frac{N+\alpha}{N+1}$, and suppose that the symbol a of the Lévy operator $\mathcal{L}$ satisfies conditions (2.4) and (2.5). If $u$ is a nonnegative solution to (1.1)-(1.3) with an initial datum satisfying $0 \leq u_{0} \in W^{1, \infty}\left(\mathbb{R}^{N}\right) \cap L^{1}\left(\mathbb{R}^{N}\right)$, then $\lim _{t \rightarrow \infty} M(t)=0$.

Again, when $q$ is greater than the critical exponent, the diffusion effects prevails for large times and, as $t \rightarrow \infty$, the mass $M(t)$ converges to a positive limit.

Theorem 2.9. Let $\lambda\langle 0, q\rangle \frac{N+\alpha}{N+1}$, and suppose that the symbol a of the Lévy operator $\mathcal{L}$ satisfies condition (2.4). If $u$ is a nonnegative solution to (1.1)-(1.3) with an initial datum satisfying $0 \leq u_{0} \in W^{1, \infty}\left(\mathbb{R}^{N}\right) \cap L^{1}\left(\mathbb{R}^{N}\right)$, then $\lim _{t \rightarrow \infty} M(t)=$ $M_{\infty}>0$.

The proof of Theorem 2.9 is based on the decay estimates of $\|\nabla u(t)\|_{p}$ proven in Theorem 3.8, below. However, as was the case for $\lambda>0$, we can significantly simplify that reasoning for Lévy operators $\mathcal{L}$ with nondegenerate Brownian part, and $q \geq 2$; see the remark following the proof of Theorem 2.9.

Our final result shows that when the mass $M(t)$ tends to a finite limit $M_{\infty}$, as $t \rightarrow \infty$, the solutions to problem (1.1)-(1.3) display a self-similar asymptotic dictated by the fundamental solution of the linear equation $u_{t}+(-\Delta)^{\alpha / 2} u=0$ which is given by the formula

$$
p_{\alpha}(x, t)=t^{-N / \alpha} p_{\alpha}\left(x t^{-1 / \alpha}, 1\right)=\frac{1}{(2 \pi)^{N / 2}} \int_{\mathbb{R}^{N}} e^{i x \xi} e^{-t|\xi|^{\alpha}} d \xi .
$$

More precisely, we have

Theorem 2.10. Let $u=u(x, t)$ be a solution to problem (1.1)-(1.3) with $u_{0} \in$ $L^{1}\left(\mathbb{R}^{N}\right) \cap W^{1, \infty}\left(\mathbb{R}^{N}\right)$, and suppose that the symbol a of the Lévy operator $\mathcal{L}$ satisfies conditions (2.4) and (2.5). If $\lim _{t \rightarrow \infty} M(t)=M_{\infty}$ exists and is finite, then

$$
\lim _{t \rightarrow \infty}\left\|u(t)-M_{\infty} p_{\alpha}(t)\right\|_{1}=0 .
$$

If, additionally,

$$
\|u(t)\|_{p} \leq C t^{-N(1-1 / p) / \alpha},
$$

for some $p \in(1, \infty]$, all $t>0$, and a constant $C$ independent of $t$, then, for every $r \in[1, p)$,

$$
\lim _{t \rightarrow \infty} t^{N(1-1 / r) / \alpha}\left\|u(t)-M_{\infty} p_{\alpha}(t)\right\|_{r}=0 .
$$

Remark 2.11. Note that, in the case $M_{\infty}=0$, the results of Theorem 2.10 only give that, as $t \rightarrow \infty,\|u(t)\|_{r}$ decays to 0 faster than $t^{-N(1-1 / r) / \alpha}$. 
Remark 2.12. For $\lambda<0$, in view of (2.12), the nonnegative solutions to (1.1)-(1.3) satisfy the estimate $0 \leq u(x, t) \leq e^{-t \mathcal{L}} u_{0}(x)$, for all $x \in \mathbb{R}^{N}$, and $t>0$. Hence, in this case, by (2.6), the decay estimate (2.16) holds true with $p=+\infty$. On the other hand, for $\lambda>0$ and sufficiently small initial data, the estimate of $\|\nabla u(t)\|_{p_{0}}$ from Theorem 3.8 applied to the integral equation (2.12) immediately implies (2.16) with $p=p_{0}$; see the statement and the proof of Theorem 3.8. In fact, following the reasoning from [3], it is possible to prove (2.16) with $p=\infty$ without any smallness assumption. That argument is based on the integral equation (2.12) and involves inequalities (2.6) and (2.11). Here, we skip other details.

\section{Existence, Uniqueness, AND MONOTONICITY}

We begin by proving that problem (1.1)-(1.3) is locally well-posed in $W^{1, \infty}\left(\mathbb{R}^{N}\right)$.

Proposition 3.1. Assume that the symbol $a=a(\xi)$ of the operator $\mathcal{L}$ satisfies condition (2.4) with some $\alpha \in(1,2]$. Then, for every $\lambda \in \mathbb{R}$, and $u_{0} \in W^{1, \infty}\left(\mathbb{R}^{N}\right)$, there exists $T=T\left(u_{0}, \lambda\right)$ such that problem (1.1)-(1.3) has a unique solution in the space $\mathcal{X}=C\left([0, T), W^{1, \infty}\left(\mathbb{R}^{N}\right)\right)$.

Proof. This result can be found in [9]. However, we want to sketch some details of the proof here because they can help in understanding the reasoning employed in the proof of Proposition 3.4, below. Our method is well-known since the local-intime solution is constructed via the mild equation (2.12) as the fixed point of the operator

$$
\mathcal{T} u(t)=e^{-t \mathcal{L}} u_{0}+\lambda \int_{0}^{t} e^{-(t-\tau) \mathcal{L}}|\nabla u(\tau)|^{q} d \tau,
$$

in the space Banach $\mathcal{X}_{T}=C\left([0, T), W^{1, \infty}\left(\mathbb{R}^{N}\right)\right)$ endowed with the norm

$$
\|u\|_{\mathcal{X}_{T}} \equiv \sup _{0 \leq t \leq T}\|u(t)\|_{\infty}+\sup _{0 \leq t \leq T}\|\nabla u(t)\|_{\infty} .
$$

Inequality (2.8), with $p=\infty$, implies

$$
\begin{aligned}
\left\|\mathcal{T} u(t)-e^{-t \mathcal{L}} u_{0}\right\|_{\infty} & \leq|\lambda| \int_{0}^{t}\left\|e^{-(t-\tau) \mathcal{L}} \mid \nabla u(\tau)\right\|^{q} d \tau \\
& \leq C|\lambda| T\left(\sup _{0 \leq \tau \leq T}\|\nabla u(\tau)\|_{\infty}\right)^{q},
\end{aligned}
$$

and, similarly (by $(2.9)$, with $p=\infty)$,

$$
\left\|\nabla \mathcal{T} u(t)-\nabla e^{-t \mathcal{L}} u_{0}\right\|_{\infty} \leq C|\lambda| T^{1-1 / \alpha}\left(\sup _{0 \leq \tau \leq T}\|\nabla u(\tau)\|_{\infty}\right)^{q}
$$

Moreover, the elementary inequality

$$
\left|a^{q}-b^{q}\right| \leq C(q)|a-b|\left(|a|^{q-1}+|b|^{q-1}\right)
$$

implies that, for each $R>0$, and for all $u, v \in \mathcal{X}_{T}$ such that $\|u\|_{\mathcal{X}_{T}} \leq R$ and $\|v\|_{\mathcal{X}_{T}} \leq R$, we have

$$
\|\mathcal{T} u-\mathcal{T} v\|_{\mathcal{X}_{T}} \leq\left(C_{1} T R^{q-1}+C_{2} T^{1-1 / \alpha} R^{q-1}\right)\|u-v\|_{\mathcal{X}_{T}} .
$$

Hence, the nonlinear operator $\mathcal{T}$ defined in (3.1) is a contraction on the ball in $\mathcal{X}_{T}$ of radius $R$ and centered at $e^{-t \mathcal{L}} u_{0}$, provided $R$ is sufficiently large and $T$ is sufficiently small. The Banach fixed point theorem guarantees the existence of a 
solution in that ball. By a standard argument involving the Gronwall lemma, this is the unique solution in the whole space $\mathcal{X}_{T}$.

In the next step, we recall a "maximum principle" which confirms the "parabolic nature" of equation (1.1) and allows us to prove inequalities (2.11).

Lemma 3.2. Let $\mathcal{L}$ be a pseudodifferential operator with the symbol $a=a(\xi)$ represented by $(2.2)$. Assume that $v \in D(\mathcal{L}) \cap C_{0}\left(\mathbb{R}^{N}\right)$ and that, for some $x_{0} \in \mathbb{R}^{N}$, we have $v\left(x_{0}\right)=\inf _{x \in \mathbb{R}^{N}} v(x) \leq 0$. Then $(\mathcal{L} v)\left(x_{0}\right) \leq 0$.

This fact is well known in the theory of generators of Feller semigroups (cf., e.g., [13, Ch. 4.5]), and we skip its simple proof. Observe that under the additional assumption, $v \in D(\mathcal{L}) \cap C^{2}\left(\mathbb{R}^{N}\right)$, it is possible to immediately deduce the result from the representation of the operator $\mathcal{L}$ given in (1.2). Indeed, for $x_{0} \in \mathbb{R}^{N}$ satisfying $v\left(x_{0}\right)=\inf _{x \in \mathbb{R}^{N}} v(x)$, we have $\nabla v\left(x_{0}\right)=0$. Hence,

$$
\mathcal{L} v\left(x_{0}\right)=-\sum_{j, k=1}^{N} Q_{j, k} \frac{\partial^{2} v\left(x_{0}\right)}{\partial x_{j} \partial x_{k}}+\int_{\mathbb{R}^{N}}\left(v\left(x_{0}\right)-v\left(x_{0}+y\right)\right) \Pi(d y) \leq 0,
$$

since $Q$ is nonnegative-definite, $v\left(x_{0}+y\right) \geq v\left(x_{0}\right)$ for all $y \in \mathbb{R}^{N}$, and the Lévy measure $\Pi(d y)$ in nonnegative (cf. also [13, Theorem 4.5.13]).

Proposition 3.3. The solution $u=u(x, t)$ constructed in Theorem 2.1 satisfies inequalities (2.11), as well as the comparison principle.

We again skip the proof because, using Lemma 3.2 (or, more generally, [9, Thm. 2 and Thm. 9]), it suffices to proceed as in [9, Thm. 3 and Prop. 2] (cf. also $[1,10,11]$ for the proof in the classical case $\mathcal{L}=-\Delta)$.

Proposition 3.4. Assume that $u_{0} \in W^{1, \infty}\left(\mathbb{R}^{N}\right)$, and $u_{0}-K \in L^{1}\left(\mathbb{R}^{N}\right)$, for a constant $K \in \mathbb{R}$. Then the solution $u=u(x, t)$ of the problem (1.1)-(1.3) constructed in Proposition 3.1 satisfies condition (2.10).

Proof. According to Remark 2.2 one can assume that $K=0$. To prove Proposition 3.4, it suffices to show that the operator $\mathcal{T}$ used in the proof of Proposition 3.1 maps the subspace of $\mathcal{X}_{T}$, defined as

$$
\mathcal{Y}_{T} \equiv\left\{u \in \mathcal{X}_{T}: \sup _{0<t \leq T}\|u(t)\|_{1}<\infty, \quad \sup _{0<t \leq T} t^{1 / \alpha}\|\nabla u(t)\|_{1}<\infty\right\},
$$

into itself.

Observe that Lévy semigroup's properties (2.6) and (2.7), with $p=1$, guarantee that $e^{-t \mathcal{L}} u_{0} \in \mathcal{Y}_{T}$, for every $u_{0} \in W^{1, \infty}\left(\mathbb{R}^{N}\right) \cap L^{1}\left(\mathbb{R}^{N}\right)$.

Now, assume that $u \in \mathcal{Y}_{T}$. It follows from the definition of the space $\mathcal{Y}_{T}$ that

$$
\begin{aligned}
& \left\|\int_{0}^{t} e^{-(t-\tau) \mathcal{L}}|\nabla u(\tau)|^{q} d \tau\right\|_{1} \\
& \quad \leq \int_{0}^{t}\|\nabla u(\tau)\|_{q}^{q} d \tau \\
& \quad \leq C T^{1-1 / \alpha} \sup _{0 \leq \tau \leq T}\|\nabla u(\tau)\|_{\infty}^{q-1} \sup _{0 \leq \tau \leq T} \tau^{1 / \alpha}\|\nabla u(\tau)\|_{1} .
\end{aligned}
$$


Moreover, by (2.7), with $p=1$, we obtain

$$
\begin{aligned}
t^{1 / \alpha} & \left\|\int_{0}^{t} \nabla e^{-(t-\tau) \mathcal{L}}|\nabla u(\tau)|^{q} d \tau\right\|_{1} \\
\leq & \leq C t^{1 / \alpha} \int_{0}^{t}(t-\tau)^{-1 / \alpha}\|\nabla u(\tau)\|_{q}^{q} d \tau \\
& \leq C T^{1-1 / \alpha} \sup _{0 \leq \tau \leq T}\|\nabla u(\tau)\|_{\infty}^{q-1} \sup _{0 \leq \tau \leq T} \tau^{1 / \alpha}\|\nabla u(\tau)\|_{1} .
\end{aligned}
$$

Hence $\mathcal{T}: \mathcal{Y}_{T} \rightarrow \mathcal{Y}_{T}$, and repeating the Banach fixed point argument as in the proof of Proposition 3.1 we show that $u \in \mathcal{Y}_{T_{1}}$, for some $T_{1} \leq T$ (and, hence, $u$ satisfies (2.10)). In order to prove that $T_{1}=T$, we proceed as follows. By Proposition 3.3, the solution satisfies inequalities (2.11). Hence, an estimate analogous to that in (3.3) combined with the (singular) Gronwall lemma implies

$$
t^{1 / \alpha}\|\nabla u(t)\|_{1} \leq C\left(T_{1},\left\|u_{0}\right\|_{1},\left\|\nabla u_{0}\right\|_{\infty}^{q-1}\right),
$$

for all $t \in\left[0, T_{1}\right]$, and a constant $C=C\left(T_{1},\left\|u_{0}\right\|_{1},\left\|\nabla u_{0}\right\|_{\infty}^{q-1}\right)$ independent of $t$. Moreover, proceeding as in (3.2) we obtain

$$
\|u(t)\|_{1} \leq\left\|u_{0}\right\|_{1}+\left\|\nabla u_{0}\right\|_{\infty}^{q-1} \int_{0}^{t}\|\nabla u(\tau)\|_{1} d \tau .
$$

Hence, it follows from (3.4) that $\sup _{t \in\left[0, T_{1}\right]}\|u(t)\|_{1}<\infty$. Consequently, by a standard reasoning, the solution exists on $\left[0, T_{1}+\varepsilon\right]$ for some $\varepsilon>0$. By the continuity argument, we finally obtain $T_{1}=T$.

Proof of Theorem 2.1. The local-in-time existence of solutions is shown in Propositions 3.1 and 3.4. Proposition 3.3 provides inequalities (2.11) and the comparison principle for $t \in[0, T]$. Finally, the standard continuity argument (like the one used at the end of the proof of Proposition 3.4) implies that $T$ can be arbitrarily large.

Given $0 \leq u_{0} \in W^{1, \infty}\left(\mathbb{R}^{N}\right) \cap L^{1}\left(\mathbb{R}^{N}\right)$, Proposition 3.4 allows us to define the "mass" of the solution to (1.1)-(1.3) by the formula

$$
M(t)=\|u(t)\|_{1}=\int_{\mathbb{R}^{N}} u(x, t) d x, \quad t \geq 0 .
$$

The following result shows the fundamental monotonicity property of this quantity.

Proposition 3.5. Assume that $u \in C\left([0, \infty), L^{1}\left(\mathbb{R}^{N}\right)\right)$ is a solution of problem (1.1)-(1.3) (or, more precisely, a solution to the integral equation (2.12)). Then, for every $t \geq 0$,

$$
M(t)=\int_{\mathbb{R}^{N}} u(x, t) d x=\int_{\mathbb{R}^{N}} u_{0}(x) d x+\lambda \int_{0}^{t} \int_{\mathbb{R}^{N}}|\nabla u(x, \tau)|^{q} d x d \tau .
$$

In particular, $M(t)$ is nonincreasing in the evaporation case, $\lambda<0$, and it is nondecreasing in the deposition case, $\lambda>0$.

Proof. Since, for every $t \geq 0, \mu^{t}$ in the representation (2.3) is a probability measure, it follows from the Fubini theorem, and from the representation (2.3), that

$$
\int_{\mathbb{R}^{N}} e^{-t \mathcal{L}} u_{0}(x) d x=\int_{\mathbb{R}^{N}} \int_{\mathbb{R}^{N}} u_{0}(x-y) \mu^{t}(d y) d x=\int_{\mathbb{R}^{N}} u_{0}(y) d y,
$$


and, similarly,

$$
\int_{\mathbb{R}^{N}} \int_{0}^{t} e^{-(t-\tau) \mathcal{L}}|\nabla u(x, \tau)|^{q} d \tau d x=\int_{0}^{t} \int_{\mathbb{R}^{N}}|\nabla u(x, \tau)|^{q} d x d \tau .
$$

Hence, identity (3.5) is immediately obtained from equation (2.12) by integrating it with respect to $x$.

We conclude this section with a result on the time-decay of certain $L^{p}$-norms of $\nabla u$ under smallness assumptions on the initial conditions. First, however, we need some auxiliary lemmata.

Lemma 3.6. Let $g:(0, \infty) \rightarrow(0, \infty)$ be a continuous function satisfying the inequality $g(t) \leq A+B g^{p}(t)$, for all $t>0$, some constants $A, B>0$, and $p>1$. If $A^{p-1} B<p^{-1}(1-1 / p)^{p-1}$, and $\limsup _{t \rightarrow 0} g(t)$ is sufficiently small, then $g(t) \leq$ $A p /(p-1)$.

Proof. A direct calculation shows that the function $f(x)=x-A-B x^{p}$ attains its maximum (for $x>0$ ) at $x_{0}=(p B)^{-1 /(p-1)}$. Moreover, $f(0)=-A<0$, and $f\left(x_{0}\right)=(p B)^{-1 /(p-1)}(1-1 / p)^{p-1}>0$, for $A^{p-1} B<p^{-1}(1-1 / p)^{p-1}$. Hence, $g(t)$ remains in the bounded connected component (containing zero) of the set $\{x \geq 0: f(x)<0\}$. Obviously, $g(t) \leq x_{0}$. However, one can improve this inequality as follows:

$$
g(t) \leq A+B g(t) g^{p-1}(t) \leq A+B g(t) x_{0}^{p-1} \leq A+g(t) / p .
$$

This completes the proof of Lemma 3.6.

Lemma 3.7. For every $p \in[1, \infty]$, there exists a constant $C$ depending only on $p$, and such that, for every $v \in L^{p}\left(\mathbb{R}^{N}\right) \cap L^{1}\left(\mathbb{R}^{N}\right)$,

$$
D(v, p) \equiv \sup _{t>0} t^{1 / \alpha}(1+t)^{\beta}\left\|\nabla e^{-t \mathcal{L}} v\right\|_{p} \leq C\left(\|v\|_{p}^{1 / \beta}+\|v\|_{1}^{1 / \beta}\right)^{\beta},
$$

where $\beta=\frac{N}{\alpha}\left(1-\frac{1}{p}\right)$.

Proof. It follows from $(2.7)$ that $\left\|\nabla e^{-t \mathcal{L}} v\right\|_{p} \leq C(t / 2)^{-1 / \alpha}\left\|e^{-(t / 2) \mathcal{L}} v\right\|_{p}$, hence, it suffices to estimate $(1+t)^{N(1-1 / p) / \alpha}\left\|e^{-t \mathcal{L}} v\right\|_{p}$. However, by inequalities (2.6), we have

$$
\begin{aligned}
& (1+t)^{N(1-1 / p) / \alpha}\left\|e^{-t \mathcal{L}} v\right\|_{p} \\
& \leq \min \left\{(1+t)^{N(1-1 / p) / \alpha}\|v\|_{p}, C(p)\left(t^{-1}+1\right)^{N(1-1 / p) / \alpha}\|v\|_{1}\right\} .
\end{aligned}
$$

The right-hand side of the above inequality, as the function of $t$, attains its maximum at $t_{0}=\left(C(p)\|v\|_{1} /\|v\|_{p}\right)^{1 / \beta}$. This completes the proof of Lemma 3.7.

Theorem 3.8. Let $\lambda \in \mathbb{R}, q>\frac{N+\alpha}{N+1}$, and suppose that the symbol a of the Lévy operator $\mathcal{L}$ satisfies (2.4), and (2.5), with a certain $\alpha \in(1,2]$. If $u=u(x, t)$ is a solution (not necessarily nonnegative) to problem (1.1)-(1.3), with the initial datum $u_{0} \in W^{1, \infty}\left(\mathbb{R}^{N}\right) \cap L^{1}\left(\mathbb{R}^{N}\right)$, then there exists an exponent $p_{0}$ satisfying conditions

$$
\frac{N+\alpha}{N+1}<p_{0}<\frac{N}{N+1-\alpha}, \quad p_{0} \leq q,
$$


and such that if $D\left(u_{0}, p_{0}\right)^{p_{0}-1}\left\|\nabla u_{0}\right\|_{\infty}^{q-p_{0}}$ is sufficiently small, then the solution $u$ satisfies the inequality

$$
t^{1 / \alpha}(1+t)^{N\left(1-1 / p_{0}\right) / \alpha}\|\nabla u(t)\|_{p_{0}} \leq C D\left(u_{0}, p_{0}\right),
$$

for all $t>0$, and a constant $C>0$ independent of $t$ and $u_{0}$.

As we shall see below, we obtain the decay estimate (3.8) for every $p_{0}$ which is sufficiently close to $(N+\alpha) /(N+1)$ and satisfies inequalities (3.7).

Proof of Theorem 3.8. Our reasoning is based on the integral equation (2.12), estimates of the semigroup $e^{-t \mathcal{L}}$ stated in (2.6)-(2.9), and several algebraic calculations on fractions. First, note that, for $\alpha>1$, we have $(N+\alpha) /(N+1)<N /(N+1-\alpha)$; hence the inequalities in (3.7) make sense.

In view of equation (2.12) and inequalities (2.6), (2.7), (2.11) we obtain

$$
\begin{aligned}
\|\nabla u(t)\|_{p_{0}} \leq & \left\|\nabla e^{-t \mathcal{L}} u_{0}\right\|_{p_{0}} \\
& +C\left\|\nabla u_{0}\right\|_{\infty}^{q-p_{0}} \int_{0}^{t}(t-\tau)^{-N\left(1-1 / p_{0}\right) / \alpha-1 / \alpha}\|\nabla u(\tau)\|_{p_{0}}^{p_{0}} d \tau .
\end{aligned}
$$

Next, we define the auxiliary function

$$
g(t)=\sup _{0 \leq \tau \leq t} \tau^{1 / \alpha}(1+\tau)^{N\left(1-1 / p_{0}\right) / \alpha}\|\nabla u(\tau)\|_{p_{0}},
$$

which, by (3.9), satisfies

$$
t^{1 / \alpha}(1+t)^{N\left(1-1 / p_{0}\right) / \alpha}\|\nabla u(t)\|_{p_{0}} \leq D\left(u_{0}, p_{0}\right)+C\left\|\nabla u_{0}\right\|_{\infty}^{q-p_{0}} g(t)^{p_{0}} h(t),
$$

for all $t>0$, and a constant $C$ independent of $t$ and $u_{0}$, and where $h$ is defined by $h(t)=t^{1 / \alpha}(1+t)^{N\left(1-1 / p_{0}\right) / \alpha} \int_{0}^{t}(t-\tau)^{-N\left(1-1 / p_{0}\right) / \alpha-1 / \alpha} \tau^{-p_{0} / \alpha}(1+\tau)^{-N\left(p_{0}-1\right) / \alpha} d \tau$.

Now, let us prove that $\sup _{t>0} h(t)<\infty$. First, note that, for every $t>0$, the integral in the definition of $h(t)$ converges because the inequality $-N\left(1-1 / p_{0}\right) / \alpha-$ $1 / \alpha>-1$ is equivalent to the condition $p_{0}<N /(N+1-\alpha)$; moreover, $-p_{0} / \alpha>-1$ since, for $\alpha \in(1,2]$, we have $p_{0}<N /(N+1-\alpha) \leq \alpha$.

For large values of $t$ the integral is bounded by $t^{\beta}$, with $\beta=-N\left(1-1 / p_{0}\right) / \alpha-$ $1 / \alpha-p_{0} / \alpha-N\left(p_{0}-1\right) / \alpha+1$; hence $h(t) \leq C t^{1-p_{0} / \alpha-N\left(p_{0}-1\right) / \alpha}$, where, for $p_{0}>$ $(N+\alpha) /(N+1)$, the exponent is negative.

Next, we analyse the behavior of $h(t)$, as $t \rightarrow 0$. In this case, say for $t \in(0,1)$, we obtain $h(t) \leq c t^{-N\left(1-1 / p_{0}\right) / \alpha-p_{0} / \alpha+1}$. Our goal is to show that

$$
\beta\left(p_{0}\right) \equiv-N\left(1-1 / p_{0}\right) / \alpha-p_{0} / \alpha+1>0,
$$

for each $p_{0}>(N+\alpha) /(N+1)$, which is sufficiently close to $(N+\alpha) /(N+1)$. This, however, follows from the continuity of the function $\beta\left(p_{0}\right)$ because, for $\alpha>1$, we have $\beta((N+\alpha) /(N+1))=N(\alpha-1)^{2} /(\alpha(N+\alpha)(N-1))>0$.

Hence, by (3.10), the function $g(t)$ satisfies the inequality

$$
g(t) \leq D\left(u_{0}, p_{0}\right)+C\left\|\nabla u_{0}\right\|_{\infty}^{q-p_{0}} g(t)^{p_{0}} .
$$

The proof is completed by an application of Lemma 3.6, because $g(t)$ is a continuous function (due to the regularity properties of the solution stated in Theorem 2.1), $\lim \sup _{t \rightarrow 0} g(t) \leq D\left(u_{0}, p_{0}\right)$, which follows from (3.10), and from the properties of function $h(t)$ shown above. 


\section{Mass evolution in the Deposition CASE}

In the deposition case, i.e., for $\lambda>0$, Proposition 3.5 asserts that the mass function $M(t)=\int_{\mathbb{R}^{N}} u(x, t) ; d x$ is nondecreasing in $t$. The next result shows that, for $q \leq(N+\alpha) /(N+1)$, as $t \rightarrow \infty$, the function $M(t)$ escapes to $+\infty$ at a certain rate, thus implying the qualitative statement of Theorem 2.3.

Theorem 4.1. Under the assumptions of Theorem 2.3 there exists $T_{0}=t_{0}\left(u_{0}\right)$ such that, for all $t \geq t_{0}\left(u_{0}\right)$, we have the following lower bounds for $M(t)$ :

(a) If $N \geq 2$, then

$$
M(t) \geq\left\{\begin{array}{cl}
C(q) \lambda M_{0}^{q} t^{(N+\alpha-(N+1) q) / \alpha}, & \text { for } 1 \leq q<\frac{N+\alpha}{N+1} \\
C(q) \lambda M_{0}^{q} \log t, & \text { for } q=\frac{N+\alpha}{N+1}
\end{array}\right.
$$

(b) If $N=1$, then

$$
M(t) \geq\left\{\begin{array}{cl}
C(q) \lambda^{1 / q} M_{0}^{2-1 / q} t^{(1+\alpha-2 q) /(2 q)}, & \text { for } 1 \leq q<\frac{1+\alpha}{2} \\
C(q) \lambda^{1 / q} M_{0}^{q-1 / q}(\log t)^{1 / q}, & \text { for } \quad q=\frac{1+\alpha}{2}
\end{array}\right.
$$

Proof. Here, we adapt the reasoning from [19]. Since $\lambda$ and $u_{0}$ are nonnegative, it follows from equality (3.5) that

$$
\lambda^{-1} M(t)=\lambda^{-1}\|u(t)\|_{1} \geq \int_{0}^{t}\|\nabla u(\tau)\|_{q}^{q} d \tau .
$$

First, consider $N \geq 2$. Note that by (2.12), with $\lambda>0$, we have $u(x, t) \geq$ $e^{-t \mathcal{L}} u_{0}(x)$, for all $(x, t) \in \mathbb{R}^{N} \times[0, \infty)$. Hence, by the Sobolev inequality, we obtain

$$
\lambda^{-1} M(t) \geq C \int_{0}^{t}\|u(\tau)\|_{N q /(N-q)}^{q} d \tau \geq C \int_{0}^{t}\left\|S(\tau) u_{0}\right\|_{N q /(N-q)}^{q} d \tau .
$$

Next, due to the assumption (2.5), we may apply Lemma 6.1 from Section 6 to show

$$
\begin{aligned}
& t^{N(1-1 / p) / \alpha}\left|\left\|S(t) u_{0}\right\|_{p}-M_{0}\left\|p_{\alpha}(\cdot, t)\right\|_{p}\right| \\
& \leq t^{N(1-1 / p) / \alpha}\left\|S(t) u_{0}-M_{0} p_{\alpha}(\cdot, t)\right\|_{p} \rightarrow 0
\end{aligned}
$$

as $t \rightarrow \infty$. Since $t^{N(1-1 / p) / \alpha}\left\|p_{\alpha}(\cdot, t)\right\|_{p}=\left\|p_{\alpha}(\cdot, 1)\right\|_{p}$ (cf. (2.14)), there exists a $t_{0}=t_{0}\left(u_{0}\right)$ such that

$$
\left\|S(\tau) u_{0}\right\|_{p} \geq \frac{1}{2} M_{0}\left\|p_{\alpha}(\cdot, 1)\right\|_{p} t^{-N(1-1 / p) / \alpha}, \quad \text { for all } \quad t \geq t_{0} .
$$

Now, we substitute this inequality, with $p=N q /(N-q)$, into (4.3) to obtain the estimate

$$
\lambda^{-1} M(t) \geq C\left(u_{0}\right) M_{0}^{q} \int_{t_{0}}^{t} \tau^{-N q(1-1 / q+1 / N) / \alpha} d \tau,
$$

which immediately implies (4.1).

The one-dimensional case requires a slightly modified argument, because the usual Sobolev embedding fails. Instead, we use the interpolation inequality

$$
\|v\|_{\infty}^{2 q-1} \leq C\|v\|_{1}^{q-1}\left\|v_{x}\right\|_{q}^{q}
$$


for $q \geq 1$, and all $v \in L^{1}(\mathbb{R})$ and $v_{x} \in L^{q}(\mathbb{R})$. Since $\|u(t)\|_{1}$ is nondecreasing (cf. Proposition 3.5), it follows from (3.5) that

$$
\begin{aligned}
\|u(t)\|_{1}^{q} & \geq \lambda\left(\int_{0}^{t}\left\|u_{x}(\tau)\right\|_{q}^{q} d \tau\right)\|u(t)\|_{1}^{q-1} \\
& \geq \lambda \int_{0}^{t}\left\|u_{x}(\tau)\right\|_{q}^{q}\|u(\tau)\|_{1}^{q-1} d \tau \\
& \geq \lambda C \int_{0}^{t}\|u(\tau)\|_{\infty}^{2 q-1} d \tau .
\end{aligned}
$$

Next, applying Lemma 6.1 as in the case $N \geq 2$, we deduce the existence of $t_{0}=$ $t_{0}\left(u_{0}\right)$, and $C>0$, such that

$$
\|u(t)\|_{\infty} \geq C M_{0} t^{-1 / \alpha}, \quad \text { for all } t \geq t_{0} .
$$

Hence, by inequality (4.5), we obtain

$$
\|u(t)\|_{1}^{q} \geq \lambda C M_{0}^{2 q-1} \int_{t_{0}}^{t} \tau^{-(2 q-1) / \alpha} d \tau
$$

which leads directly to (4.2).

At this point we are ready to provide proofs of Theorems 2.4 and 2.6.

Proof of Theorem 2.4. Combining the interpolation inequality

$$
\|v\|_{p} \leq C(p)\|v\|_{1}^{\frac{N+p}{(N+1) p}}\|\nabla v\|_{\infty}^{\frac{N(p-1)}{(N+1) p}}
$$

valid for each $p \in[1, \infty]$, and all $v \in W^{1, \infty}\left(\mathbb{R}^{N}\right) \cap L^{1}\left(\mathbb{R}^{N}\right)$, with estimate (3.6), we see that the quantity $D\left(u_{0}, p_{0}\right)$ from Theorem 3.8 can be controlled from above by a quantity depending only on $\left\|u_{0}\right\|_{1}$ and $\left\|\nabla u_{0}\right\|_{\infty}$. Hence, for small either $\left\|u_{0}\right\|_{1}$ or $\left\|\nabla u_{0}\right\|_{\infty}$, the smallness assumption required in Theorem 3.8 is satisfied.

Next, the decay estimates obtained in Theorem 3.8 allow us to prove that $|\nabla u|^{q} \in L^{1}\left(\mathbb{R}^{N} \times[0, \infty)\right)$, which immediately implies $M_{\infty}<\infty$. Indeed, choosing $p_{0}$ satisfying conditions from Theorem 3.8, the required integrability property of $\nabla u$ follows from the following inequalities:

$$
\begin{aligned}
\int_{0}^{\infty} \int_{\mathbb{R}^{N}}|\nabla u(x, \tau)|^{q} d x d \tau & \leq\left\|u_{0}\right\|_{\infty}^{q-p_{0}} \int_{0}^{\infty}\|\nabla u(\tau)\|_{p_{0}}^{p_{0}} d \tau \\
& \leq C\left\|u_{0}\right\|_{\infty}^{q-p_{0}} \int_{0}^{\infty} \tau^{-p_{0} / \alpha}(1+\tau)^{-N\left(p_{0}-1\right) / \alpha} d \tau<\infty
\end{aligned}
$$

because the condition $-p_{0} / \alpha-N\left(p_{0}-1\right) / \alpha<-1$ is automatically satisfied for $p_{0}>(N+\alpha) /(N+1)$.

Proof of Theorem 2.6. We have already mentioned in the Introduction that, by a linear change of variables, the Lévy operator can be written in the form

$$
\mathcal{L}=-\Delta+\mathcal{H},
$$

where $\mathcal{H}$ is another Lévy operator given by the integral part in (2.2). We also recall that each Lévy operator $\mathcal{H}$ is positive in the sense that, for every $p \geq 1$ and 
$u \in D(\mathcal{H})$, it satisfies the inequality

$$
\int_{\mathbb{R}^{N}}(\mathcal{H} u)\left(|u|^{p-1} \operatorname{sign} u\right) d x \geq 0 .
$$

For the proof of (4.6), we refer the reader to [13, Ch. 4.6].

In order to prove that $M_{\infty}<\infty$, it suffices to show that $|\nabla u|^{q} \in L^{1}\left(\mathbb{R}^{N} \times[0, \infty)\right)$. However, due to the inequality

$$
\|\nabla u(t)\|_{q}^{q} \leq\left\|\nabla u_{0}\right\|_{\infty}^{q-2}\|\nabla u(t)\|_{2}^{2},
$$

which is a direct consequence of (2.11), we only need to prove that

$$
\sup _{t>0} \int_{0}^{t} \int_{\mathbb{R}^{N}}|\nabla u(x, \tau)|^{2} d x d \tau<\infty .
$$

To this end, we multiply equation (1.1) by $u^{p}$ and, integrating by parts, obtain

$$
\begin{aligned}
& p \int_{0}^{t} \int_{\mathbb{R}^{N}} u^{p-1}|\nabla u|^{2} d x d \tau+\int_{0}^{t} \int_{\mathbb{R}^{N}} u^{p} \mathcal{H} u d x d \tau \\
& =\frac{1}{p+1} \int_{\mathbb{R}^{N}}\left(u_{0}^{p+1}-u(t)^{p+1}\right) d x+\lambda \int_{0}^{t} \int_{\mathbb{R}^{N}} u^{p}|\nabla u|^{q} d x d \tau .
\end{aligned}
$$

The second term on the left-hand side of (4.7) is nonnegative by inequality (4.6) (with $p-1$ replaced by $p$ ). Hence

$$
\int_{0}^{t} \int_{\mathbb{R}^{N}} u^{p-1}|\nabla u|^{2} d x d \tau \leq \frac{\left\|u_{0}\right\|_{p+1}^{p+1}}{p(p+1)}+\frac{b}{p} \int_{0}^{t} \int_{\mathbb{R}^{N}} u^{p}|\nabla u|^{2} d x d \tau,
$$

with $b=\lambda\left\|\nabla u_{0}\right\|_{\infty}^{q-2}$.

From now on, our reasoning is similar to that presented in [19]. We claim that, for every integer $k \geq 1$,

$$
\int_{0}^{t} \int_{\mathbb{R}^{N}}|\nabla u|^{2} d x d \tau \leq \sum_{\ell=1}^{k} \frac{\left\|u_{0}\right\|_{\ell+1}^{\ell+1} b^{\ell-1}}{(\ell+1) !}+\frac{b^{k}}{k !} \int_{0}^{t} \int_{\mathbb{R}^{N}} u^{k}|\nabla u|^{2} d x d \tau .
$$

Indeed, for $k=1$, this is just inequality (4.8), with $p=1$. To show (4.9) for $k>1$ it is sufficient to proceed by induction:

Choose $k_{0}$ large enough so that

$$
\frac{b^{k_{0}}\left\|u_{0}\right\|_{\infty}^{k_{0}}}{k_{0} !} \leq \frac{1}{2} .
$$

Hence, inequality (4.9) with $k=k_{0}$ implies the estimate

$$
\int_{0}^{t} \int_{\mathbb{R}^{N}}|\nabla u|^{2} d x d \tau \leq 2 \sum_{\ell=1}^{k_{0}} \frac{\left\|u_{0}\right\|_{\ell+1}^{\ell+1} b^{\ell-1}}{(\ell+1) !}
$$

which completes the proof of Theorem 2.6.

\section{Mass eVolution in the EVAPORAtion CASE}

In this section, we study equation (1.1) in the evaporation case, i.e., for $\lambda<0$. In view of Proposition 3.5, the mass function $M(t)$ is now a decreasing function of $t$. Our goal is to find out under what conditions it remains bounded away from zero or, alternatively, when it vanishes at infinity, i.e., when $M_{\infty}=\lim _{t \rightarrow \infty} M(t)=$ $\lim _{t \rightarrow \infty} \int_{\mathbb{R}^{N}} u(x, t) d x=0$. We begin by some auxiliary results. 
Lemma 5.1. If $w \in W^{1,1}\left(\mathbb{R}^{N}\right)$, then, for every $R>0$,

$$
\|w\|_{1} \leq 2 R \int_{|x| \leq 3 R}|\nabla w(x)| d x+2 \int_{|x|>R}|w(x)| d x .
$$

A short and elementary proof of Lemma 5.1 can be found in the paper by BenArtzi and Koch [3].

Lemma 5.2. Let $\lambda<0$. Assume that the symbol $a=a(\xi)$ of the Lévy operator $\mathcal{L}$ given by the formula (2.2) satisfies the assumptions (2.4) and (2.5). If $r \in C(0, \infty)$ is a nonnegative function such that

$$
\lim _{t \rightarrow \infty} r(t) t^{-1 / \alpha}=+\infty
$$

then

$$
\lim _{t \rightarrow \infty} \int_{|x| \geq r(t)} u(x, t) d x=0 .
$$

Proof. Since $\lambda<0$, it follows from the integral equation (2.12) that, for all $t \geq 0$, and $x \in \mathbb{R}^{N}$, we have $0 \leq u(x, t) \leq e^{-t \mathcal{L}} u_{0}(x)$. Hence

$$
\begin{aligned}
\int_{|x| \geq r(t)} u(x, t) d x \leq & \int_{|x| \geq r(t)} e^{-t \mathcal{L}} u_{0}(x) d x \\
\leq & \int_{|x| \geq r(t)}\left|e^{-t \mathcal{L}} u_{0}(x)-\left\|u_{0}\right\|_{1} p_{\alpha}(x, t)\right| d x \\
& +\left\|u_{0}\right\|_{1} \int_{|x| \geq r(t)} p_{\alpha}(x, t) d x .
\end{aligned}
$$

As $t \rightarrow \infty$, the first term on the right-hand side tends to 0 by Lemma 6.1 with $p=1$. In the second term, we change the variables $y=x t^{-1 / \alpha}$ to obtain

$$
\int_{|x| \geq r(t)} p_{\alpha}(x, t) d x=\int_{|y| \geq r(t) t^{-1 / \alpha}} p_{\alpha}(y, 1) d y \rightarrow 0, \quad \text { as } \quad t \rightarrow \infty,
$$

in view of the self-similarity of the form $p_{\alpha}(x, t)=t^{-N / \alpha} p_{\alpha}\left(x t^{-1 / \alpha}, 1\right)$, the assumption $(5.1)$, and since $p_{\alpha}(\cdot, 1) \in L^{1}\left(\mathbb{R}^{N}\right)$.

Now we are ready to prove the results of Section 2 describing mass evolution in the evaporation case.

Proof of Theorem 2.8. Since $M(t)$ is nonnegative, equation (3.5) with $\lambda<0$ implies that

$$
\int_{0}^{\infty} \int_{\mathbb{R}^{N}}|\nabla u(x, \tau)|^{q} d x d \tau \leq\left\|u_{0}\right\|_{1}
$$

For $t \geq 0$, define

$$
\omega(t)=\left(\int_{t / 2}^{\infty} \int_{\mathbb{R}^{N}}|\nabla u(x, \tau)|^{q} d x d \tau\right)^{1 / q},
$$

and notice that $\omega=\omega(t)$ is a nonincreasing function on $[0, \infty)$ which satisfies the condition

$$
\lim _{t \rightarrow \infty} \omega(t)=0
$$


Now, for $t \geq 1, s \in(t / 2, t)$, and $R>0$, we infer from Lemma 5.1 combined with the Hölder inequality that

$$
\|u(s)\|_{1} \leq C R^{1+N(1-1 / q)}\|\nabla u(s)\|_{q}+2 \int_{|x|>R}|u(x, s)| d x .
$$

Since $s \mapsto\|u(s)\|_{1}$ is nonincreasing on $(t / 2, t)$, it follows from inequality (5.3), and the Hölder inequality, that

$$
\begin{aligned}
\|u(t)\|_{1} & \leq \frac{2}{t} \int_{t / 2}^{t}\|u(s)\|_{1} d s \\
& \leq C R^{1+N(1-1 / q)} t^{-1 / q} \omega(t)+\frac{4}{t} \int_{t / 2}^{t} \int_{|x|>R}|u(x, s)| d x d s .
\end{aligned}
$$

Next, fix $\delta \in\left(0,(1+N(1-1 / q))^{-1}\right)$ and define

$$
R(t)=t^{1 / \alpha} \omega(t)^{-\delta}
$$

This function is nondecreasing (because $\omega(t)$ is nonincreasing) which implies that $R(t) \geq R(s)$ for all $s \in[t / 2, t]$. Hence, substituting $R=R(t)$ into inequality (5.4) we obtain

$$
\begin{aligned}
\|u(t)\|_{1} \leq & C t^{(1+N(1-1 / q)) / \alpha-1 / q} \omega(t)^{1-\delta(1+N(1-1 / q))} \\
& +\frac{4}{t} \int_{t / 2}^{t} \int_{|x|>R(s)}|u(x, s)| d x d s .
\end{aligned}
$$

The first term on the right-hand side tends to zero, as $t \rightarrow \infty$, because the inequality $(1+N(1-1 / q)) / \alpha-1 / q \leq 0$ is equivalent to $q \leq(N+\alpha) /(N+1)$ and $\omega(t)^{1-\delta(1+N(1-1 / q))} \rightarrow 0$. The second term converges to zero by the Lebesgue Dominated Convergence theorem, and Lemma 5.2, because

$$
\frac{4}{t} \int_{t / 2}^{t} \int_{|x|>R}|u(x, s)| d x d s=4 \int_{1 / 2}^{1} \int_{|x|>R(t \tau)}|u(x, t \tau)| d x d \tau .
$$

Hence $M(t)=\|u(t)\|_{1} \rightarrow 0$, as $t \rightarrow \infty$, and the proof is complete.

Proof of Theorem 2.9. For $\varepsilon \in(0,1]$, we denote by $u^{\varepsilon}=u^{\varepsilon}(x, t)$ the solution to (1.1)-(1.3) with $\varepsilon u_{0}(x)$ as the initial datum. Since, by the comparison principle from Theorem 2.1, $0 \leq u^{\varepsilon}(x, t) \leq u(x, t)$, for all $x \in \mathbb{R}^{N}$, and $t>0$, it suffices to show that, for small $\varepsilon>0$,

$$
\lim _{t \rightarrow \infty} M^{\varepsilon}(t)=\lim _{t \rightarrow \infty} \int_{\mathbb{R}^{N}} u^{\varepsilon}(x, t) d x=M_{\infty}^{\varepsilon}>0 .
$$

However, by Proposition 3.5,

$$
M_{\infty}^{\varepsilon}=\varepsilon \int_{\mathbb{R}^{N}} u_{0}(x) d x-|\lambda| \int_{0}^{\infty} \int_{\mathbb{R}^{N}}\left|\nabla u^{\varepsilon}(x, \tau)\right|^{q} d \tau .
$$


Hence, for sufficiently small $\varepsilon>0$, and for $p_{0}$ satisfying the assumptions of Theorem 3.8 , we have

$$
\begin{aligned}
M_{\infty}^{\varepsilon} \geq & \varepsilon \int_{\mathbb{R}^{N}} u_{0}(x) d x \\
& -|\lambda|\left\|\nabla \varepsilon u_{0}\right\|_{\infty}^{q-p_{0}} \int_{0}^{\infty}\left\|\nabla u^{\varepsilon}(\tau)\right\|_{p_{0}}^{p_{0}} d \tau \\
\geq & \varepsilon \int_{\mathbb{R}^{N}} u_{0}(x) d x \\
& -|\lambda| C\left\|\nabla \varepsilon u_{0}\right\|_{\infty}^{q-p_{0}} D\left(\varepsilon u_{0}, p_{0}\right)^{p_{0}} \int_{0}^{\infty} \tau^{-p_{0} / \alpha}(1+\tau)^{-N\left(p_{0}-1\right) / \alpha} d \tau .
\end{aligned}
$$

We have already shown in the proof of Theorem 3.8 that the integral on the righthand side is finite. Moreover, by the definition of $D\left(u_{0}, p_{0}\right)$, we have $D\left(\varepsilon u_{0}, p_{0}\right)=$ $\varepsilon D\left(u_{0}, p_{0}\right)$ and, consequently,

$$
M_{\infty}^{\varepsilon} \geq \varepsilon \int_{\mathbb{R}^{N}} u_{0}(x)-\varepsilon^{q}|\lambda|\left\|\nabla u_{0}\right\|_{\infty}^{q-p_{0}} D\left(u_{0}, p_{0}\right) C,
$$

with $C>0$ independent of $\varepsilon$, and $u_{0}$. Since $q>1$, it follows from (5.5) that, for sufficiently small $\varepsilon>0$, necessarily $M_{\infty}^{\varepsilon}>0$.

Remark 5.3. If we strengthen the assumptions in Theorem 2.9, and demand that $q \geq 2$ and $\mathcal{L}$ has a nondegenerate Brownian part, i.e. $\mathcal{L}=\Delta+\mathcal{H}$ (cf. the proof of Theorem 2.6), then multiplying equation (1.1) by $u$ and integrating over $\mathbb{R}^{N} \times[0, t]$, we obtain

$$
\begin{aligned}
& 2 \int_{0}^{t} \int_{\mathbb{R}^{N}}|\nabla u|^{2} d x d \tau+\int_{0}^{t} \int_{\mathbb{R}^{N}} u \mathcal{H} u d x d \tau \\
& =\frac{1}{2} \int_{\mathbb{R}^{N}}\left(u_{0}^{2}-u(t)^{2}\right) d x-|\lambda| \int_{0}^{t} \int_{\mathbb{R}^{N}} u|\nabla u|^{q} d x d \tau .
\end{aligned}
$$

In particular, we have (cf. (4.6) and (4.7))

$$
\int_{0}^{t} \int_{\mathbb{R}^{N}}|\nabla u|^{2} d x d \tau \leq \frac{1}{2}\left\|u_{0}\right\|_{2}^{2} .
$$

Hence, repeating the reasoning from the proof of Theorem 2.9, we obtain, for sufficiently small $\varepsilon>0$, that

$$
M_{\infty}^{\varepsilon} \geq \varepsilon \int_{\mathbb{R}^{N}} u_{0}(x) d x-\varepsilon^{q}(|\lambda| / 2)\left\|\nabla u_{0}\right\|_{\infty}^{q-2}\left\|u_{0}\right\|_{2}^{2}>0 .
$$

Note that, in this case, we do not need decay estimates from Theorem 3.8.

\section{Self-Similar ASYMPtotics}

Assumptions (2.4) and (2.5) allow us to approximate $e^{-t \mathcal{L}} u_{0}$ by a multiplicity of the kernel

$$
p_{\alpha}(x, t)=\frac{1}{(2 \pi)^{-N / 2}} \int_{\mathbb{R}^{N}} e^{i x \xi} e^{-t|\xi|^{\alpha}} d \xi .
$$

Indeed, we have the following. 
Lemma 6.1. If the symbol $a(\xi)$ of the Lévy operator $\mathcal{L}$ satisfies conditions (2.4) and (2.5), then, for each $p \in[1, \infty]$, and $u_{0} \in L^{1}\left(\mathbb{R}^{n}\right)$,

$$
\lim _{t \rightarrow \infty} t^{N(1-1 / p) / \alpha}\left\|e^{-t \mathcal{L}} u_{0}-\int_{\mathbb{R}^{N}} u_{0}(x) d x p_{\alpha}(t)\right\|_{p}=0 .
$$

Proof. This result is immediately obtained from the inequality

$$
\left\|h * g(\cdot)-\left(\int_{\mathbb{R}^{n}} h(x) d x\right) g(\cdot)\right\|_{p} \leq C\|\nabla g\|_{p}\|h\|_{L^{1}\left(\mathbb{R}^{n},|x| d x\right)},
$$

which is valid for each $p \in[1, \infty]$, all $h \in L^{1}\left(\mathbb{R}^{n},|x| d x\right)$, and every $g \in C^{1}\left(\mathbb{R}^{n}\right) \cap$ $W^{1,1}\left(\mathbb{R}^{n}\right)$, with a constant $C=C_{p}$ independent of $g$ and $h$. The inequality itself is a simple consequence of the Taylor expansion.

To prove the lemma we apply (6.2), with $h=e^{-t \mathcal{K}} u_{0}$, and $g(x)=p_{\alpha}(x, t)$, assuming first that $u_{0} \in L^{1}\left(\mathbb{R}^{n},|x| d x\right)$. The general case of $u_{0} \in L^{1}\left(\mathbb{R}^{n}\right)$ can then be handled by an approximation argument. Details of such a reasoning can be found in [8, Cor. 2.1 and 2.2].

Now, we are in a position to prove the final, main theorem of Section 2.

Proof of Theorem 2.10. Since $M_{\infty}$ is finite, formula (3.5) for $M(t)$ implies that $\|\nabla u\|_{q}^{q} \in L^{1}(0, \infty)$. It follows from the integral equation (2.12) that

$$
\left\|u(t)-e^{-\left(t-t_{0}\right) \mathcal{L}} u\left(t_{0}\right)\right\|_{1} \leq \int_{t_{0}}^{t}\|\nabla u(\tau)\|_{q}^{q} d \tau, \quad \text { for all } t \geq t_{0} \geq 0 .
$$

Hence,

$$
\begin{aligned}
\left\|u(t)-M_{\infty} p_{\alpha}(t)\right\|_{1} \leq & \int_{t_{0}}^{t}\|\nabla u(\tau)\|_{q}^{q} d \tau \\
& +\left\|e^{-\left(t-t_{0}\right) \mathcal{L}_{2}} u\left(t_{0}\right)-M\left(t_{0}\right) p_{\alpha}(t)\right\|_{1} \\
& +\left\|p_{\alpha}(t)\right\|_{1}\left|M\left(t_{0}\right)-M_{\infty}\right| .
\end{aligned}
$$

Letting $t \rightarrow \infty$, and using Lemma 6.1 for the second term on the right-hand side, we obtain

$$
\limsup _{t \rightarrow \infty}\left\|u(t)-M_{\infty} p_{\alpha}(t)\right\|_{1} \leq \int_{t_{0}}^{\infty}\|\nabla u(\tau)\|_{q}^{q} d \tau+\left|M\left(t_{0}\right)-M_{\infty}\right|,
$$

because $\left\|p_{\alpha}(t)\right\|_{1}=1$. Since $t_{0}$ can be arbitrarily large, and each term on the right-hand side tends to 0 , as $t_{0} \rightarrow \infty$, the proof of (2.15) is complete.

Now, we apply the Hölder inequality to obtain

$$
\left\|u(t)-M_{\infty} p_{\alpha}(t)\right\|_{r} \leq\left\|u(t)-M_{\infty} p_{\alpha}(t)\right\|_{1}^{1-\gamma}\left(\|u(t)\|_{p}^{\gamma}+M_{\infty}\left\|p_{\alpha}(t)\right\|_{p}^{\gamma}\right),
$$

with $\gamma=(1-1 / r) /(1-1 / p)$. Finally, to prove (2.17), apply inequality (2.16), the asymptotic result $(2.15)$, and the identity $\left\|p_{\alpha}(t)\right\|_{p}=t^{-N(1-1 / p) / \alpha}\left\|p_{\alpha}(1)\right\|_{p}$.

\section{REFERENCES}

1. L. Amour and M. Ben-Artzi, Global existence and decay for viscous Hamilton-Jacobi equations, Nonlinear Anal. 31 (1998), 621-628. MR1487850 (99c:35098)

2. S. Benachour, G. Karch and Ph. Laurençot, Asymptotic profiles of solutions to viscous Hamilton-Jacobi equations, J. Math. Pures Appl. 83 (2004), 1275-1308. MR2092308 (2005g:35146) 
3. M. Ben-Artzi and H. Koch, Decay of mass for a semilinear parabolic equation, Comm. Partial Differential Equations 24 (1999), 869-881. MR1680909 (2000a:35098)

4. M. Ben-Artzi, Ph. Souplet and F.B. Weissler, The local theory for viscous Hamilton-Jacobi equations in Lebesgue spaces, J. Math. Pures Appl. 81 (2002), 343-378. MR1967353 (2004a:35100)

5. P. Biler, T. Funaki, and W.A. Woyczynski, Fractal Burgers equation, J. Differential Equations 148 (1998), 9-46. MR1637513 (99g:35111)

6. P. Biler, G. Karch, and W.A. Woyczyński, Asymptotics for multifractal conservation laws, Studia Math. 135 (1999), 231-252. MR1708995 (2000h:35098)

7. P. Biler, G. Karch, and W.A. Woyczyński, Asymptotics for conservation laws involving Lévy diffusion generators, Studia Math. 148 (2001), 171-192. MR1881259 (2002j:60140)

8. P. Biler, G. Karch, and W.A. Woyczyński, Critical nonlinearity exponent and self-similar asymptotics for Lévy conservation laws, Ann. I.H. Poincaré - Analyse non linéaire, 18 (2001), 613-637. MR1849690 (2002f:35035)

9. J. Droniou and C. Imbert, Fractal first order partial differential equations, Arch. Rational Mech. Anal. 182 (2006), 299-331. MR2259335

10. B. Gilding, The Cauchy problem for $u_{t}=\Delta u+|\nabla u|^{q}$, large-time behaviour, J. Math. Pures Appl. 84 (2005), 753-785. MR2138640 (2006a:35149)

11. B. Gilding, M. Guedda and R. Kersner, The Cauchy problem for $u_{t}=\Delta u+|\nabla u|^{q}$, J. Math. Anal. Appl. 284 (2003), 733-755. MR1998665 (2005h:35165)

12. C. Imbert, A non-local regularization of first order Hamilton-Jacobi equations, J. Differential Equations 211 (2005), 218-246. MR2121115 (2006e:35038)

13. N. Jacob, Pseudo-differential operators and Markov processes. Vol. I. Fourier analysis and semigroups. Imperial College Press, London, 2001. MR1873235 (2003a:47104)

14. E.R. Jakobsen, K.H. Karlsen, Continuous dependence estimates for viscosity solutions of integro-PDEs, J. Differential Equations 212 (2005), 278-318. MR2129093 (2005k:35198)

15. E.R. Jakobsen, K.H. Karlsen, A "maximum principle for semicontinuous functions" applicable to integro-partial differential equations, NoDEA Nonlinear Differential Equations Appl. 13 (2006), no. 2, 137-165. MR2243708 (2007c:45020)

16. B. Jourdain, S. Méléard, and W.A. Woyczynski, Probabilistic approximation and inviscid limits for one-dimensional fractional conservation laws, Bernoulli 11 (2005), 689-714. MR2158256 (2006e:60094)

17. B. Jourdain, S. Méléard, and W.A. Woyczynski, A probabilistic approach for nonlinear equations involving the fractional Laplacian and a singular operator, Potential Analysis 23 (2005), 55-81. MR2136209 (2006a:35327)

18. M. Kardar, G. Parisi, Y.-C. Zhang, Dynamic scaling of growing interfaces, Phys. Rev. Lett. 56 (1986), 889-892.

19. $\mathrm{Ph}$. Laurençot and $\mathrm{Ph}$. Souplet, On the growth of mass for a viscous Hamilton-Jacobi equation, J. Anal. Math. 89 (2003), 367-383. MR1981925 (2004c:35188)

20. J.A. Mann, Jr., W.A. Woyczynski, Growing fractal interfaces in the presence of self-similar hopping surface diffusion, Physica A. Statistical Physics 291 (2001), 159-183.

21. W.A. Woyczynski, Burgers-KPZ Turbulence - Göttingen Lectures, Lecture Notes in Mathematics 1700, Springer-Verlag 1998. MR1732301 (2000j:60077)

Instytut Matematyczny, Uniwersytet WrocŁaWski, Pl. Grunwaldzki 2/4, 50-384 WrocŁaW, Poland

E-mail address: karch@math.uni.wroc.pl

$U R L:$ http://www.math.uni.wroc.pl/ karch

Department of Statistics and the Center for Stochastic and Chaotic Processes in Science and Technology, Case Western Reserve University, Cleveland, Ohio, 441067054

E-mail address: waw@po.cwru.edu

URL: http://stat.cwru.edu/Wojbor/ 